\title{
La narrativa como herramienta docente dentro de la gamificación de la estadística en el Grado en Turismo
}

\author{
Jesús Barreal \\ jbarreal@ucm.es \\ Universidad Complutense de Madrid, España
}

\section{Gil Jannes}

gjannes@ucm.es

Universidad Complutense de Madrid, España

\begin{abstract}
Resumen
La gamificación consiste en ludificar los contenidos de una determinada materia o asignatura a través de dinámicas, mecánicas y componentes. Con este proceso se puede lograr una mayor motivación e implicación del alumno, así como fomentar el desarrollo de competencias y del aprendizaje autónomo. El presente trabajo describe el proceso de implementación de esta herramienta para la asignatura "Estadística Aplicada al Sector Turístico" del Grado en Turismo con el objetivo de lograr presentar un proceso formativo dinámico y atractivo para el alumno. No obstante, se elabora desde un punto transversal que permita su implementación en asignaturas similares de enseñanzas superiores de grado. En más detalle, se desarrolla una narrativa entorno a la promoción profesional del alumno dentro de una empresa hotelera ficticia donde irá alcanzando mayor rango profesional a medida que avanza el juego y va superando una serie de pruebas/eventos. Esta narrativa fomenta el interés intrínseco en la actividad por parte del alumno, al mismo tiempo que refuerza la utilidad del propio juego de cara a futuras experiencias profesionales.
\end{abstract}

\section{Palabras clave}

Gamificación; Grado en Turismo; Métodos estadísticos; Narrativa; proceso formativo. 


\title{
Narrative as a teaching tool in the gamification of statistics in the Degree in Tourism
}

\author{
Jesús Barreal \\ jbarreal@ucm.es \\ Universidad Complutense de Madrid, Spain \\ Gil Jannes \\ gjannes@ucm.es \\ Universidad Complutense de Madrid, Spain
}

\begin{abstract}
Gamification consists in modifying the contents of a certain subject or course in a gamelike manner through dynamics, mechanics and components. Through this process, a greater motivation and involvement of the student can be achieved, while also encouraging the development of certain skills as well as autonomous learning. This paper describes the process of implementing a gamification tool for the subject "Statistics Applied to the Tourism Sector" of the Degree in Tourism with the aim of presenting a dynamic and attractive training process for the student. The tool is developed from a transversal point of view which allows its implementation in similar subjects of higher education. Concretely, a narrative is developed around the professional promotion of the student within a fictitious hotel company where (s)he will reach a higher professional rank as the game progresses and (s)he passes a series of tests and/or events. This narrative encourages the student's intrinsic interest in the activity, while reinforcing the usefulness of the game itself for future professional experiences.
\end{abstract}

\section{Keywords}

Gamification; Degree in Tourism; Statistical methods; Narrative; Formative process 


\section{Introducción}

La innovación docente se presenta cada vez más como un campo de trabajo imprescindible que tiene el profesorado para adaptar las necesidades de sus clases al perfil del alumnado. Entre sus metodologías se encuentra la gamificación o ludificación, que consiste en adaptar las dinámicas de los juegos para aumentar la implicación y participación del alumnado (Cortizo et al., 2011). Esta actividad es transversal a los distintos niveles docentes e incluso aplicable al ámbito empresarial. Efectivamente, la gamificación se ha aplicado en niveles educativos tanto primarios, secundarios como universitarios (Salcu y Acatrinei, 2013; Hofacker et al, 2016; Contreras, 2016), así como también a ámbitos empresariales para formar y entrenar a sus empleados (Gómez y de Pablos, 2013; Rodríguez et al., 2016; Miguel et al., 2017). Centrándose en la docencia universitaria, la gamificación es una herramienta incipiente en los procesos de aprendizaje que pretende dinamizar y motivar a los alumnos (Martínez y Pérez, 2015; Prieto, Díaz, Monserrat y Reyes, 2014). Por otro lado, esta metodología se puede emplear tanto a toda la asignatura como a contenidos puntuales. Esto permite distinguir entre la gamificación estructural y la superficial.

La gamificación no se debe confundir con la mera adaptación de juegos a los contenidos docentes, sino que se debe de utilizar para desarrollar incentivos intrínsecos del alumno (Kokkalia et al, 2017). Esta afirmación surge de la Teoría de la Autodeterminación propuesta por Ryan y Deci (2000) en la que propone que los individuos persiguen actividades que les ayuden a satisfacer necesidades intrínsecas, como la autonomía o adquisición de competencias.

En el contexto actual, las herramientas informáticas han avanzado mucho, lo que ha favorecido su inclusión en distintos aspectos de la estructura académica. En este sentido, se observa cómo se ha implementado el uso del aula virtual o de la elaboración de prácticas en soportes informáticos y/o virtuales. Todo esto ha conferido al sistema universitario de mayor versatilidad y adaptación a las necesidades formativas de los alumnos. Este hecho se refleja en las aulas a través de presentaciones dinámicas, en soportes como Power Point o Prezit, o actividades interactivas con aplicaciones como Kahoot o Socrative. Estas últimas utilidades han instaurado el juego como un sistema de aprendizaje a distintos niveles. Por tanto, también, se ha puesto de manifiesto su utilidad para las enseñanzas universitarias.

Por otra parte, en la actualidad, el alumnado forma parte de una generación inmersa en un mundo digitalizado y demanda la aplicación de herramientas dinámicas que favorezcan su proceso de aprendizaje. Así, si le unimos lo anterior, se plantea la opción de realizar una serie de juegos mediante el empleo de las nuevas tecnologías para cubrir las necesidades y aspiraciones docentes del alumno.

El instrumento educativo de gamificación consiste en elaborar una serie de actividades interactivas entre el alumno y un entorno virtual en el que se le plantean problemáticas relacionadas con la temática de la asignatura. A mayores, en ellas se expondrán una serie de cuestiones reales que el alumno debe resolver a través de los contenidos teóricos que previamente se han expuesto en clase. Esto se puede realizar de manera individual o en grupos de alumnos. La idoneidad de cada una de las modalidades dependerá de las situaciones planteadas y de las metodologías necesarias para resolverlas. Cabe mencionar que las actividades grupales también favorecen el desarrollo de otras aptitudes como la comunicación o el trabajo en equipo. Este tipo de actividades se enmarca en la actual línea de mejora educativa que proponen diversas publicaciones (Castilla, Romana y López, 2013). Éstas plantean que la gamificación de los cuestionarios es un importante elemento de innovación y dinamización docente en el aula.

Ante esta casuística se exponen una serie de objetivos para desarrollar esta nueva metodología. La principal finalidad de este trabajo docente es el de proponer herramientas dinámicas que aumenten la implicación del alumnado y en particular su participación, comunicación y trabajo en equipo. 
Para ello se desarrollará este marco a la asignatura de estadística aplicada al sector turístico para favor favorecer el aprendizaje en competencias del alumno. Una componente importante en este marco es que la gamificación, más que un juego como tal, debe también contribuir a la percepción de aplicabilidad de los contenidos de la asignatura a situaciones reales que favorezcan la toma de decisiones turísticas. A tal efecto, se hará hincapié en el desarrollo de una narrativa de fondo relacionada con el sector turístico, con el objetivo de captar el interés intrínseco del estudiante. De tal modo, aunque inevitablemente parte de la motivación será extrínseca (la consecución de puntos, insignias y otro tipo de premios), pretendemos evitar que este interés sea de corta duración y se vaya apagando una vez la emoción inicial de la obtención de premios disminuya. Por último, este diseño se espera que pueda ser replicable para otros grados y asignaturas relacionadas.

Partiendo de todo lo anterior, los autores han enfocado su gamificación en la asignatura "Estadística Aplicada al Sector Turístico" del primer curso del Grado en Turismo, pues es la única materia de todo el plan de estudios de este grado que está relacionada con los métodos cuantitativos. Además, cabe destacar, que la mayor parte del alumnado que accede al Grado en Turismo procede del Bachiller en Humanidades o Ciencias Sociales (Pavía, Martínez, Morillas y García, 2013). Por tanto, existe por parte del estudiantado una percepción de falta de relevancia de este tipo de asignaturas y un bajo nivel de implicación (Cárdenas, Pulido y Carrillo, 2016). Esto dificulta su motivación en la adquisición de competencias relacionadas con los métodos estadísticos. Así, además de los argumentos generales expuestos anteriormente, existen razones concretas relacionadas con el carácter de la asignatura y del alumnado que hacen necesario la realización de actividades dinámicas que incrementen sus estímulos y le animen a participar de manera activa en el proceso de aprendizaje. Hasta donde los presentes autores sepan, el proyecto presentado a continuación supone el primer programa de implementación de una estrategia sistemática de gamificación en una asignatura de corte cuantitativo en las enseñanzas universitarias españolas del turismo.

Con todo esto, el presente trabajo se estructura de la siguiente forma. Se inicia con una breve revisión bibliográfica en el que se describen algunos trabajos previos relacionados con los puntos clave de la gamificación. Posteriormente se expondrá la metodología empleada en el diseño del proceso gamificador teniendo en cuenta el epígrafe anterior. Después de este paso se diseña el juego a través de los conceptos claves anteriormente descritos, con particular énfasis en la narrativa. Finalmente se concluye el texto con una descripción de algunos aspectos concretos de la implementación prevista para este proyecto, así como de una serie de futuras opciones para mejorar el trabajo realizado y fomentar su aplicación a otros ámbitos.

\section{Revisión bibliográfica}

Numerosos investigadores han realizado investigaciones sobre la gamificación. En este sentido Mora et al. (2015) ha realizado una revisión de un gran número de ellas estudiando su grado de viabilidad económica, la lógica del juego, la medida de su eficiencia o calidad, las implicaciones psicológicas o su interactividad. Así observan que la lógica e interactividad del juego son factores muy importantes a la hora de desarrollarlo. Los autores también remarcan la importancia de emplear métricas que permitan adaptar la gamificación a las necesidades docentes, aunque concluyen que en la literatura no existe una fórmula homogénea entre los estudios actuales. Por otro lado, Hamari et al. (2014) destacan que los estudios actuales hacen hincapié en que esta metodología aumenta la competencia, lo que sugiere que debe ser un factor a considerar en su planteamiento. Del mismo modo inciden en la dificultad que supone el diseño de juego y la evaluación de sus tareas. Por tanto, sugieren que todos ellos deben ser factores a considerarse en 
un proceso de gamificación docente.

Desde el punto de vista psicológico, trabajos como el de Martínez y Moral (2015), O'Donovan et al. (2013) o Fitz-Walter et al. (2011) remarcan la labor motivacional de la gamificación en los alumnos. Así, a través del juego se logra una mayor implicación del alumno en la asignatura, hecho que se alcanza mediante las dinámicas que este recurso ofrece al proceso de aprendizaje. En este sentido Richter et al. (2015), siguiendo la teoría de autorrealización, destaca que los juegos presentan tres componentes que se corresponden a otras tantas necesidades de los jugadores. Estos elementos son los extrínsecos, basados en las teorías de las necesidades, como la pirámide de Maslow o la teoría de la autoeficacia; los sociales, derivados de la teoría de la inversión personal (TIP) o la comparación social; y los extrínsecos, provenientes de la teoría del valor esperado o del refuerzo de Skinner. Partiendo de estas premisas, los autores establecen una serie de incentivos o recompensas y sus funciones dentro de las dinámicas motivacionales de la gamificación en el aula.

\begin{tabular}{|c|c|c|}
\hline TEORÍA MOTIVADORA & INCENTIVOS/RECOMPENSAS & FUNCIONES \\
\hline \multirow{2}{*}{ Autoeficacia } & $\begin{array}{l}\text { Efecto audio / verbal / visual / } \\
\text { música / sonidos }\end{array}$ & Retroalimentación \\
\hline & Barra de progreso & Retroalimentación, logros \\
\hline \multirow{2}{*}{$\begin{array}{l}\text { Autoeficacia, establecimiento } \\
\text { de objetivos, TIP, valor de } \\
\text { expectativa, logros necesarios }\end{array}$} & Puntos / bonificación / dividendo & $\begin{array}{l}\text { Retroalimentación, } \\
\text { recompensa, estado, } \\
\text { logros, competencia, } \\
\text { progresión, propiedad. }\end{array}$ \\
\hline & Minijuegos / retos / misiones & $\begin{array}{l}\text { Recompensa, estado, } \\
\text { competencia, logros }\end{array}$ \\
\hline \multirow{4}{*}{$\begin{array}{l}\text { Autoeficacia, establecimiento } \\
\text { de objetivos, TIP, valor de } \\
\text { expectativa, comparación } \\
\text { social }\end{array}$} & Insignias & $\begin{array}{l}\text { Estado y reputación, } \\
\text { logros y logros pasados, } \\
\text { colección, competencia, } \\
\text { propiedad }\end{array}$ \\
\hline & Activos virtuales & $\begin{array}{l}\text { Recompensa, social, } \\
\text { estatus, logros, } \\
\text { propiedad, expresiones de } \\
\text { personalidad }\end{array}$ \\
\hline & Tabla clasificatoria & $\begin{array}{l}\text { Estado y reputación, } \\
\text { logros, competencia }\end{array}$ \\
\hline & $\begin{array}{l}\text { Recompensa por elección de } \\
\text { colores, potencia... }\end{array}$ & Logros \\
\hline $\begin{array}{l}\text { Autoeficacia, establecimiento } \\
\text { de objetivos, TIP, valor de }\end{array}$ & Logros & $\begin{array}{l}\text { Colección, estado, } \\
\text { competición, }\end{array}$ \\
\hline
\end{tabular}




\begin{tabular}{|l|l|l|}
\hline \begin{tabular}{l} 
expectativa, logro de $\begin{array}{l}\text { necesidades, comparación } \\
\text { social }\end{array}$ \\
\cline { 2 - 3 }
\end{tabular} & Niveles & $\begin{array}{l}\text { descubrimiento, } \\
\text { progresión. }\end{array}$ \\
\hline $\begin{array}{l}\text { Comparación social, teoría de } \\
\text { la inversión personal, valor de } \\
\text { expectativa. }\end{array}$ & Avatar & $\begin{array}{l}\text { Retroalimentación, estado } \\
\text { y reputación, logros, } \\
\text { competencia, desafío } \\
\text { moderado. }\end{array}$ \\
\hline
\end{tabular}

Tabla 1. Bases teóricas para establecer incentivos y refuerzos

Fuente: Richter et al (2015)

Por otro lado, muchos autores hacen hincapié en el diseño de una narrativa o relato para implicar y motivar al alumno (Kapp, 2012; Batle y González, 2017; Rodríguez et al., 2018). Es decir, recomiendan dotar una historia a la gamificación que incentive al alumno a participar en ella. Según Kapp et al. (2013) la creación esta parte del proceso es importante siempre que se "establezca un desafío concreto que implique un mínimo de curiosidad en los participantes por realizar las actividades". Para diseñar este relato, se puede usar como hilo constructivo el estudio semiótico de Todorov (1969) que propone que la narrativa se edifica desde cinco preceptos:

1. Iniciar la historia: presentar los personajes, el espacio de desarrollo de la actividad, los antecedentes y el tiempo de desarrollo.

2. El desarrollo: la evolución de los hechos y acciones del juego que mantengan la intriga o complicación: se establece una progresión de incidentes y episodios que complican la acción y mantienen la intriga

3. La valoración: se evalúan los sucesos del juego, tanto por el narrador como por los jugadores.

4. El desenlace: concluye la acción a través de la resolución del conflicto.

5. La situación final: a través de los anteriores episodios se llega a un nuevo estado resultante de las acciones llevadas a cabo.

Autores como Werbach y Hunter (2015) proponen que se han de establecer una serie de dinámicas para gamificar contenidos docentes. Así, el proceso debe de tener restricciones, emociones, narrativa, evolución y relaciones. En este mismo sentido, González (2016) establece que el diseño debe de organizarse teniendo en cuenta, entre otros, la problemática, las reglas, los eventos y los resultados de la gamificación. Marache y Brangier (2013) consideran esencial que el alumno debe de poder elegir libremente si participa o no en el juego, por lo tanto, debe de ser flexible la entrada y salida de jugadores. Además, según estos autores, los beneficios de la gamificación deben de estar bien explicados para que el alumno perciba su importancia en su proceso formativo. En caso contrario implicará una desmotivación y un posible abandono del juego por parte del estudiante. La personalización es otro factor a tener en cuenta, pues se deben de generar diferentes perfiles dependiendo de la tipología de los participantes. Esto causará que estén más involucrados en la actividad al verse identificado en ella. La duración de la gamificación debe de presentar iteraciones a largo plazo, esto es, no debe de centrarse en un momento puntual, sino que debe de involucrar una planificación más larga. También se deben de eliminar todos los efectos secundarios no deseados, como pueden ser el exceso de presión o la sensación de pérdida de eficiencia. Por último, los autores proponen que se tenga en cuenta el contexto legal y ético para que no se incurran en injerencias en datos privados o que no se tenga en cuenta los intereses de los 
alumnos.

Para el diseño de la estructura y tareas de las actividades gamificadas, Contador (2015) propone que deben de seguir la definición de "competición saludable" propuesta por Yu et al. (2002) y Shindler (2007). Siguiendo a estos autores, determina que la competición ha de presentar características como, por ejemplo:

- Las temáticas y tareas han de ser dinámicas ofreciendo un gran número de alternativas al alumno.

- $\quad$ El alumno ha de tener la sensación de que puede alterar su posición en el ranking a través de su puntuación.

- $\quad$ El trabajo ha de ser repartido homogéneamente entre los participantes.

- Se ha de activar las habilidades sociales de los alumnos.

- Se ha de controlar la implicación y actividad de cada estudiante.

Por otro lado, el comportamiento de los jugadores no es homogéneo. Así, Tondello et al (2016) establece seis diferentes tipos de participantes en la gamificación que presentan distintas percepciones y sensibilidades dentro del juego. Esta tipología y sus características se pueden resumir en la Figura 1.

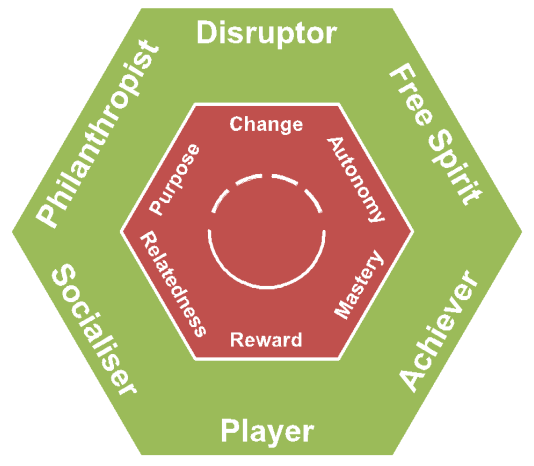

Figura. 1. Tipos de usuarios de la gamificación Fuente: Tondello et al (2016)

Partiendo de la anterior figura, los autores definen los tipos de jugadores y sus características como:

- Filántropos, buscan realizar actividades sin esperar nada a cambio. De tal manera que para gamificar una asignatura se debe de establecer actividades en las que se comparta el conocimiento tanto dentro del grupo como para el resto de los participantes sin ninguna compensación a cambio.

- Socializadores, su intención es interactuar con sus compañeros y generar conexiones sociales. Para este perfil se deben de realizar actividades que incentivan la interacción entre los estudiantes. Esto se puede lograr a través del trabajo compartido o la propia competición siempre que se fomenten las relaciones personales y la transmisión de habilidades o conocimientos

- Espíritus libres, están motivados por la autonomía e independencia de explorar por ellos mismos. En este sentido este perfil demandará la exploración de problemas de la vida real y plantear variedad de soluciones es una de las dinámicas que mejor se adaptan para los alumnos exploradores. Además, la gamificación debe de ser dinámica, con contenido desbloqueable, creativa y personificable. 
- Exitosos, la competitividad supone una motivación y buscan desarrollarse a nivel personal. Este jugador se sentirá más motivado si tiene que resolver problemas relativamente complejos.

- Jugadores, buscan la recompensa independientemente de la actividad desarrollada. La generación de un sistema de recompensas es fundamental para este tipo de alumno. En este sentido debe explicarse correctamente el sistema de puntuación y su implicación en el diseño de los rankings.

- Reaccionarios, tienden a llevar el juego al límite, buscan cambios y cuestionan el sistema. Para ellos se debe de implementar un sistema de seguimiento del desarrollo del juego en el que se tenga en cuenta la opinión del alumno. En este sentido se deben de crear plataformas innovativas o de voto electrónico que recojan las sensibilidades de los alumnos.

Atendiendo a esta clasificación, los autores proponen generar estrategias para integrar las sensibilidades de los diferentes tipos de jugadores y así garantizar un correcto diseño de la actividad.

Siguiendo a Cantador (2015), también se deben de establecer una serie de dinámicas generales para mejorar la implicación del alumno. En este sentido propone que se debe de ofrecer al alumno una continua información del progreso formativo y retroalimentación en todas las tareas planteadas. Por tanto, el participante debe de conocer el resultado obtenido en cada una de ellas y su resolución. Además, también sugiere que durante el desarrollo el alumno debe estar intrigado acerca de la futura evolución del juego y sentir cierta presión temporal para finalizar las tareas individuales iniciadas en el aula.

Los elementos de la estructura del juego son diversos, pero autores como Martínez y Moral (2015) proponen distinguir, basándose en los juegos convencionales, entre sus dinámicas y sus mecánicas. Las primeras incluyen los logros, la competición y la expresión, mientras que las segundas la misión, los niveles, los desafíos, los puntos y la clasificación. Dentro de las mecánicas del juego se pueden implementar cuestionarios de respuestas simultáneas a través de plataformas virtuales de uso libre o de pulsadores (Rodríguez, 2017). Por su parte, los autores Cantador y Conde (2010) diseñan un proceso formativo en equipo a través de la resolución de cuestionarios. Así, como se ve en la Figura 2, se observa cómo a partir de unos grupos se pueden establecer pautas para recompensar en un ranking la resolución de cuestionarios.

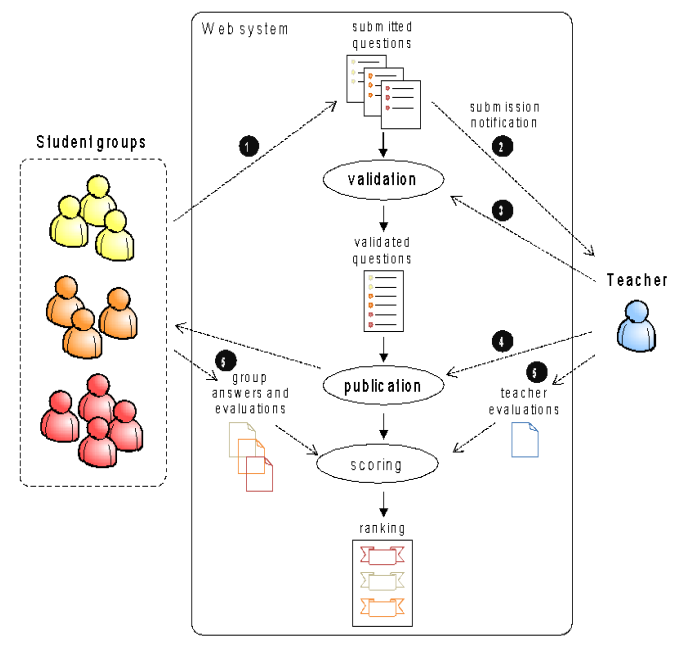

Figura. 2. Diagrama de trabajo en equipo

Fuente: Cantador y Conde (2010) 
Una de las opciones en las que se puede aplicar la gamificación es en el aprendizaje basado en problemas. Esto consiste en diseñar juegos o actividades en las que se plantean una serie de cuestiones o problemas que el alumno debe resolver según su criterio, cálculos o razonamiento siguiendo las pautas establecidas previamente para participar en la gamificación (Cantador, 2015). Con esto el alumno adquiere competencias como la resolución eficiente de problemas reales, aprendizaje autónomo, capacidad de planificación y trabajo en equipo.

\section{Metodología}

Como se ha observado en la sección anterior, existen múltiples fórmulas para gamificar una asignatura. Este trabajo tendrá en cuenta las principales aportaciones descritas anteriormente. Así, tomará en cuenta la propuesta de González (2016) que subraya la importancia de dotar de una estructura planificada para lograr una correcta implementación e implicación del alumnado. Además, siguiendo a Rodríguez et al. (2018), se considerará la construcción de la narrativa como hilo principal de la estructura de la gamificación y se implicarán las propuestas respecto a las mecánicas de juego propuestas por Martínez y Moral (2015). Para ello se establece que el diseño debe de organizarse de acuerdo a la Figura 3:
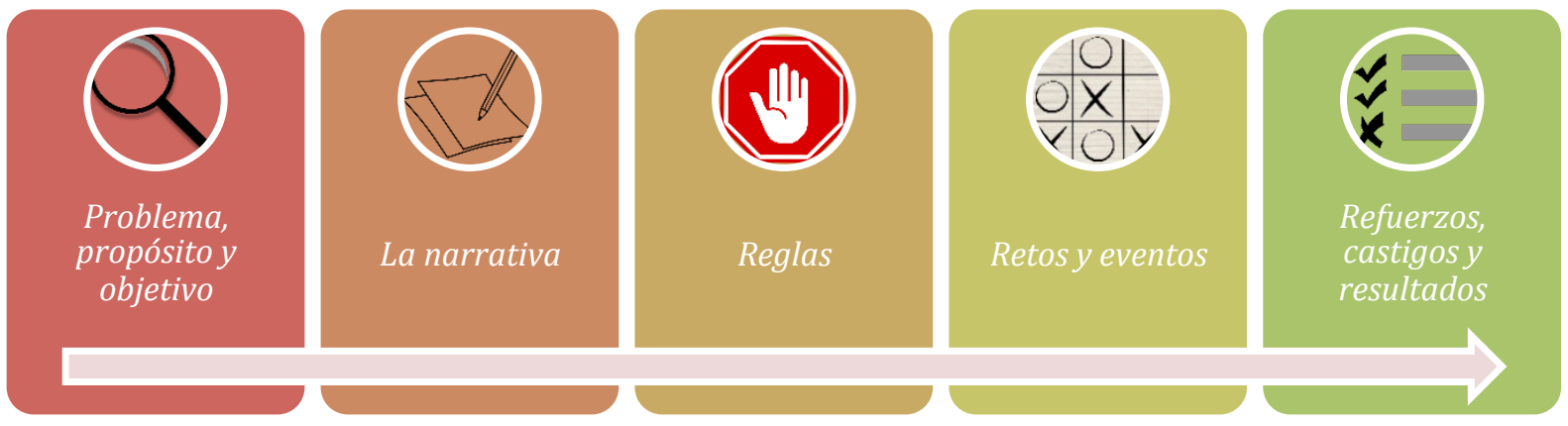

Figura. 3. Diseño de metodología

Fuente: Elaboración propia a partir de la bibliografía anterior

- Problema, propósito y objetivo: en este apartado se acotará y definirá la problemática, así como el alcance de su aplicación. A partir de lo anterior se relatan los objetivos y el modo de conseguirlos.

- La narrativa: se ambienta el juego de acuerdo a una serie de parámetros para lograr una mayor motivación e implicación por parte del alumno. Será la razón lógica que guiará al alumno a lo largo de las fases del juego y será a través de una casuística real que permita al alumno identificarse dentro de la historia.

- Reglas: se establecerán una serie de condiciones para participar, así como para poder abandonar el juego. Estas reglas tendrán en cuenta los distintos grupos de jugadores que la literatura aporta y han de ser acatadas por todos los miembros.

- Retos y eventos: se diseñará un cronograma con las actividades a realizar, aunque no todas visibles para el alumno. En este caso se hará referencia a los ejercicios o casos que el alumno deberá resolver, tanto individualmente como en grupo, para ir superando las fases.

- Refuerzos, castigos y resultados: Tras la resolución de las actividades planteadas se ofrecerá al alumno la posibilidad de consultar sus errores. Además, se establecerá un sistema de incentivos para que el alumno pueda ver su evolución formativa. Finalmente, se le expondrán los participantes mecanismos para conocer su valoración del juego. 
En todas las fases anteriores se considerarán los distintos tipos de jugadores que suelen participar en la gamificación. Por tanto, se tendrán en cuenta dichas sensibilidades en toda la formulación del juego y evitar el abandono o el rechazo a participar. Esto implica que el diseño ha de ser dinámico y flexible para que cada tipo de jugador pueda satisfacer sus necesidades individuales. Esto no supone que se abandone el objetivo principal, sino que se debe de diseñar los puntos anteriores en sentido amplio y con una retroalimentación para evitar el desánimo entre los participantes. A continuación, se describe en detalle los diferentes elementos de la confección del juego, con particular énfasis en la narrativa, cuyo objetivo es el fomento del interés intrínseco del alumnado.

\section{Diseño}

\section{a. Problema, propósito y objetivo}

Para iniciar el proceso de gamificación se debe de determinar cuál es el razonamiento por el que es importante implementar esta acción docente. En el caso de la asignatura mencionada se debe al poco incentivo que tienen los alumnos para implicarse en la asignatura, pues es la única de estadística, y en sentido más amplio: la única asignatura de corte matemático, en todo el Grado. Por tanto, la actividad planteada tiene como propósito involucrar al alumno en la asignatura y que logre una mayor comprensión de la utilidad futura de los conceptos adquiridos.

La asignatura de "Estadística Aplicada al Sector Turístico" presenta cuatro bloques claramente diferenciados. El primero es una introducción general en la que se contextualiza al alumno la estadística para explicarle su nacimiento y evolución, así como las utilidades que de ella puede obtener. En la segunda fase se describen las distintas herramientas que la estadística descriptiva ofrece para analizar los datos de una variable, su concentración y su relación con otros datos. Seguidamente se estudian aplicaciones para calcular la probabilidad de una variable, tanto para modelos discretos como continuos. Para finalizar se le ofrece al alumno las técnicas de estimación de parámetros, intervalos de confianza y contraste de hipótesis. Dadas las características de la asignatura, la gamifación se puede aplicar a toda la asignatura o individualmente a cada una de las partes.

La asignatura establece que el alumno debe de adquirir las competencias generales de CG1 Capacidad de análisis y síntesis; CG2 Comunicación oral y escrita en lengua nativa; CG3 Resolución de problemas; y CG4 Razonamiento crítico. Mientras que también se establecen dos competencias específicas: CE5 Aplicar metodología de investigación para resolver los problemas empíricos que se plantean en el ámbito del turismo; y CE20 Analizar y utilizar las tecnologías de la información y las comunicaciones (TIC) en los distintos ámbitos del sector turístico.

Con esto, el objetivo de la gamificación es incentivar el alumno a alcanzar las competencias establecidas en la asignatura a través de juegos no lúdicos que les propongan la resolución de casos reales. Para lograr este objetivo se deben de establecer juegos dinámicos y flexibles que involucren al alumno y que presenten múltiples formas de interacción en el aula.

\section{b. Narrativa}

La historia que se le plantea al alumno parte de la idea de que ha terminado sus estudios y se ha insertado en el mercado laboral. Entonces comienza a trabajar de aprendiz en la recepción de una cadena hotelera en la que espera ir subiendo en el escalafón laboral en función de su calificación. El proyecto se presenta bajo el nombre de GERENTUR, nombre de la cadena hotelera virtual, aunque la narrativa también se puede ambientar en otros apartados turísticos como la gestión de museos o de parques naturales. Se estipula que en la cadena hotelera existen cinco niveles 
laborales: Recepcionista, Jefe de Servicio, Director de Hotel, Gerente de Área de Negocio y miembro del Consejo de Administración. Así, en la Figura 4 se puede ver la pirámide por la que se desplazará el alumno a lo largo del juego.

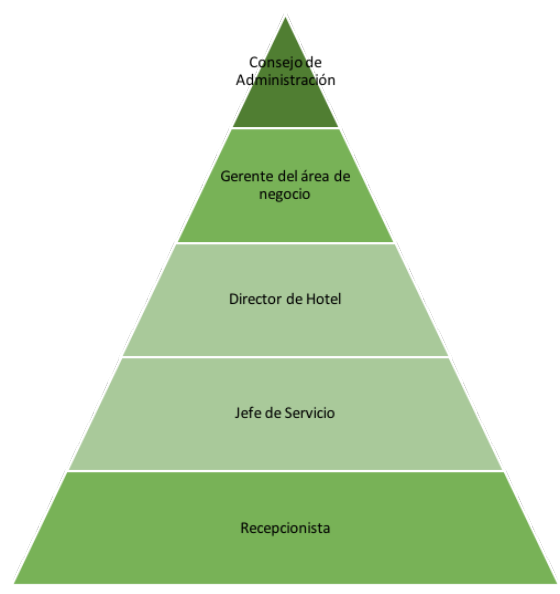

Figura. 4. Escala laboral de la cadena Hotelera Fuente: Elaboración propia

Partiendo de esta dinámica, el alumno sabe que puede ir subiendo en la escala si va resolviendo correctamente problemas y situaciones a los que se podría enfrentar si tuviera esa escala laboral. Por tanto, se involucra al estudiante en sus futuras expectativas laborales, así como en las problemáticas que puedan enfrentar en el futuro, y que están relacionadas con los aspectos de la Estadística que abordamos en la asignatura. Esto ayuda a que reconozcan la utilidad de las herramientas que les ofrece la asignatura y vean sus aplicaciones reales. El proceso de gamificación supondrá para el participante un constante crecimiento de nivel en el aula. De esta manera está estructurado que el alumno aumente de posición jerárquica a medida que vaya superando diversas pruebas de conocimiento y aptitudes.

A grandes rasgos, la evolución del juego se divide en dos partes: una parte individual, que corresponde con los tres primeros puestos profesionales - Recepcionista, Jefe de Servicio, y Director de Hotel; y una fase grupal, que corresponde con los dos niveles superiores - Gerente de Área de Negocio y miembro del Consejo de Administración. Todos los alumnos que participan en el juego pasan a la fase grupal en el mismo momento, y también pasan de Gerente de Área de Negocio a Consejo de Administración en el mismo momento. La evolución dentro de la fase individual, sin embargo, es personal y depende de la puntuación obtenida en la misma por el alumno.

Así, en función del nivel alcanzado en la primera y de la puntuación concreta que hayan obtenido, cada alumno tendrá prioridad para elegir el perfil que les corresponderá durante la fase grupal. Concretamente, dentro de los escalafones grupales que son el de Gerente de Área de Negocio y el de miembro del Consejo de Administración, los perfiles que pueden ser escogidos son los siguientes:

- En la fase de Gerente de Área, se formarán grupos de 3 integrantes, con los siguientes perfiles:

- Coordinador: es el encargado de repartir las tareas específicas a realizar entre los integrantes del grupo y realizar el informe de trabajo. También tiene la responsabilidad de notificar al profesor en caso de ausencia o falta de participación sistemática por parte de algún integrante del equipo.

- Portavoz: Es el encargado de hablar en público y exponer la resolución del problema, si ésta fuera requerida. 
Polivalente/vocal: se encarga de calcular los resultados e interpretarlos junto al resto de los integrantes. Como habilidad, esta figura puede ocupar cualquiera de los puestos anteriores siempre que se ausente alguno de los integrantes del grupo.

- En la fase de Consejo de Administración, los grupos tendrán 6 integrantes, y además de las figuras anteriores, se crea un perfil adicional:

- Secretario General: se encarga de la redacción final del informe en el que plantean la resolución del problema.

Los grupos se constituirán por los profesores organizadores de forma equilibrada. Es decir que, en la fase de Gerente de Área, cada grupo tendrá un integrante que haya terminado en el primer tercio en la fase individual, uno del segundo tercio, y uno del último tercio. En la fase de Consejo de Administración, se fusionarán dos grupos de Gerente de Área.

La jerarquización descrita tiene por tanto el múltiple objetivo de reflejar la realidad laboral, además de motivar al alumno a progresar en el juego y de motivarle a obtener una buena puntuación. Esto último sucede debido a que dentro de cada fase deberá de obtener puntuaciones altas para tener prioridad en el momento de escoger el perfil concreto de su preferencia. Finalmente, las puntuaciones alcanzadas contribuirán a la nota que el alumno obtenga en la evaluación continua de la asignatura.

\section{c. Reglas}

El alumno debe de consentir expresamente a través de un documento marco que acepta las reglas del juego. En caso contrario, deberá realizar todas las actividades de manera individual y será calificado por la metodología tradicional de resolución de ejercicios. De tal manera que el participante deberá aceptar las siguientes condiciones:

- Descargar las plataformas virtuales necesarias para el desarrollo de la gamificación.

- Trabajar en equipo con los compañeros asignados.

- Respetar los turnos de respuesta.

- Realizar las tareas asignadas en función del rol que se ha atribuido.

- Acatar los sistemas de puntuación y penalización establecidos.

- Aceptar la publicación de las puntuaciones, así como la publicación en el Aula Virtual de la asignatura de las mejores tareas realizadas.

- Ante cualquier conflicto acudir al profesor para informarle, y aceptar la autoridad del profesor y las decisiones que este pueda tomar para resolver cualquier situación de duda o conflicto.

- Cumplir con los códigos éticos institucionales.

- Aceptar la actividad como herramienta de evaluación continua.

\section{d. Retos y eventos}

Tal como se describió anteriormente, inicialmente el juego es individual para posteriormente formar equipos en función de la calificación obtenida individualmente. En la primera fase se trata de realizar cuestionarios para ir adquiriendo puntos por las respuestas correctas e ir subiendo en el escalafón laboral de la empresa hotelera. Por esta vía se puede alcanzar hasta la figura de Director de Hotel. En concreto, se establecen intervalos de puntuación en el que si el alumno alcanza 50 puntos pasa de Recepcionista a Jefe de Servicio, y si alcanza otros 75 más pasa a figurar como Director de Hotel. En esta fase se emplearán dos tipos de actividades: resolución de ejercicios a través de cálculos exactos y preguntas de respuesta múltiple. Ambos se realizarán a través de 
aplicaciones informáticas como Kahoot, Socrative o Aula Virtual. En la segunda fase, se componen una serie de grupos en función de la puntuación obtenida previamente, que seguirán las especificaciones detalladas en las secciones anteriores, y cada uno de ellos tendrán que resolver una serie de estudios de caso en función de una serie de problemáticas.

La primera fase de la gamificación afectará a los primeros temas de la asignatura, esto es, a la introducción general y a la estadística descriptiva. El alumno tendrá que resolver cuestiones como las que se plantean en la Figura 5. En el ejemplo se exponen varias opciones acerca de una definición y el alumno tendrá que seleccionar la correcta. También se pueden plantear ejercicios sencillos en los que el alumno obtiene la respuesta a través de un cálculo directo, por ejemplo, de la varianza de una serie de datos.

Cuáles de las siguientes medidas NO se ven afectadas ante un cambio de escala:
a. Media
b. Coeficiente de variación
c. Desviación típica
d. Mediana

Figura. 5. Ejemplo de práctica individual Fuente: Elaboración propia

Por su parte, la segunda fase afecta a los temas relacionados con la probabilidad de una variable, técnicas de estimación de parámetros, intervalos de confianza y contraste de hipótesis. En este caso se le expondrá al grupo de alumnos un proyecto que deberá de resolver, y respecto al cual deberá de realizar un informe y/o exponer públicamente su resolución. Un ejemplo de esta fase lo encontramos en la Figura 6, donde se expone un caso práctico global para todos los grupos.

Ultramarinos Miranda conocen los datos de ganancias diarias y clientes que se exponen a continuación:

\begin{tabular}{|l|l|}
\hline \multicolumn{1}{|c|}{ Clientes $(X)$} & \multicolumn{1}{|c|}{ Ganancias $(Y)$} \\
\hline 41 & $422,53 €$ \\
\hline 77 & $747,83 €$ \\
\hline 61 & $623,35 €$ \\
\hline 21 & $212,96 €$ \\
\hline 68 & $703,76 €$ \\
\hline 22 & $168,01 €$ \\
\hline 49 & $488,20 €$ \\
\hline 62 & $617,98 €$ \\
\hline 39 & $379,76 €$ \\
\hline 79 & $761,15 €$ \\
\hline
\end{tabular}

Figura 6. Base de datos diaria de número de clientes y las ganancias asociadas a un establecimiento. Fuente: Elaboración propia

El gerente del establecimiento quiere conocer la relación entre ambas variables y obtener una forma funcional para relacionar sus ganancias con el número de clientes que efectúan compras en su ultramarinos. Por lo tanto, el gerente se pone en contacto con vuestra asesoría para que le remitan un informe en el que se le explique dicha relación y poder 
prever los beneficios de los días en los que espera que vayan 45, 50 y 55 clientes.

Con esta información el alumno deberá realizar un pequeño informe estadístico en el que relate la exposición del problema, las herramientas que considera oportunas para resolverlo, los cálculos realizados y las conclusiones alcanzadas. También se puede requerir su exposición al grupo que mejor resuelva el caso, para fomentar una demostración de originalidad y un intercambio de conocimientos entre alumnos.

\section{e. Refuerzos, castigos y resultados}

En la resolución de las actividades irá parejo a un sistema de puntuación, que variará en función de si la actividad es individual o colectiva. En el primer caso, se puntuará positivamente las respuestas correctas y su celeridad en su respuesta. De tal manera que se establecerá una clasificación en función de las respuestas correctas emitidas, y en caso de empate se dilucidará la ordenación en función del que fuera más rápido en contestar. A medida que se vayan finalizando las rondas el alumno tendrá un puesto asignado y una puntuación global. Dicho valor será el que determine a que escalón pertenece cuando llegue a la segunda fase del juego. Al comenzar la segunda fase, los integrantes de cada grupo podrán elegir su rol en orden de la puntuación que cada uno haya obtenido en la fase individual. Durante esta segunda fase, a la puntuación individual de cada alumno se sumará la puntuación obtenida en las actividades colectivas del grupo al que pertenezca. En este apartado la puntuación irá en función de los criterios habituales, como:

- La claridad en la exposición de la resolución de ejercicios, tanto oral como escrita.

- El empleo correcto del lenguaje estadístico.

- La identificación correcta de las herramientas necesarias para resolver el caso.

- Presentación en tiempo y forma de los documentos.

- Planificación ordenada y adecuada para la ejecución de los retos a los que se enfrente cada grupo.

En ambas fases el alumno irá recibiendo una retroalimentación de sus respuestas. Esto es, una vez concluida cada actividad el alumno percibirá el solucionario tanto de los cuestionarios como de los casos prácticos. Así, todos los participantes podrán autoevaluar su proceso de aprendizaje y mejorar en aquellos apartados en los que han obtenido peores puntuaciones. Además, en algunos casos prácticos, los grupos tendrán que supervisarse entre ellos para detectar errores o inconsistencias con lo aprendido en todo el curso. En función de dichas anotaciones se seleccionará a los mejores y lo expondrán en clase. Con todo esto, al final del curso, el alumno tendrá una puntuación que se corresponderá con una posición en la clasificación final. A partir de ese valor el alumno podrá conocer su calificación obtenida en el apartado práctico de la asignatura.

Por otro lado, los alumnos deberán entregar un documento de sesiones, en el que se establezca la figura asignada a cada miembro del grupo, el reparto de trabajo, su grado de cumplimiento y la fecha de ejecución. Este documento debe ir firmado y acatado por todos los integrantes y sin el mismo no se evaluará la resolución del caso entregado. Pues así se tiene constancia de qué se ha realizado grupalmente y se conoce qué actividades realizó cada integrante. También es un elemento de control para la toma de decisiones con aquellos alumnos que decidan no cooperar con el resto de integrantes del grupo. 


\section{Implementación}

La primera aplicación del juego deberá realizarse sobre uno o más grupos de la asignatura, dejando por lo menos uno de ellos como grupo de control. Esta distinción docente se realiza con el fin de evaluar la idoneidad docente de esta dinámica gamificadora y poder comparar los resultados académicos obtenidos con o sin ella. Así, se puede obtener conclusiones robustas a cerca de la capacidad motivacional de la asignatura gamificada dentro del perfil de cada uno de los grados universitarios. Pues siguiendo Hanus y Fox (2015) remarcan que se debe de estudiar analíticamente cada proceso gamificador para cuantificar si resulta motivador para el alumno. Esto se deberá realizar los primeros años de implantación, para posteriormente seguir con controles de calidad específicos vía cuestionario, reuniones con alumnos o intercambios de percepciones en el aula.

Paralelamente al estudio analítico se debe de establecer un cronograma para desarrollar el juego. Así, inicialmente, se va a llevar a cabo una breve sesión para dar a conocer al alumno la historia, las reglas y los retos de la asignatura gamificada. A mayores, se le dará más información a través una sección dentro del Aula Virtual o de un portal web en el que podrán realizar consultas en caso de que tengan dudas fuera de las sesiones regulares. De tal manera como se observa en la Figura 7 , en dicho recurso web el alumno tendrá acceso a la historia, reglas o sistema de puntuaciones de la actividad gamificada, entre otros contenidos útiles que favorezcan a la comprensión del juego.

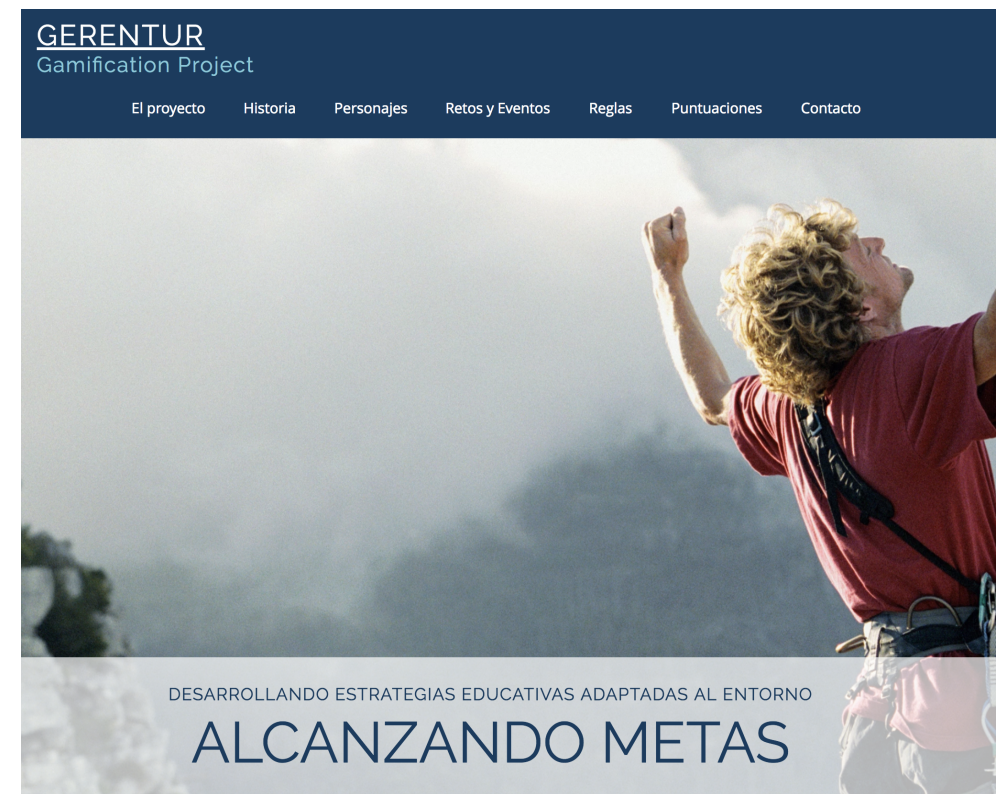

Figura. 7. Ejemplo de Página web del contenido gamificado Fuente: Elaboración propia

En las sucesivas clases posteriores se desarrollará el juego en las secciones de la asignatura en las que se deban de resolver ejercicios, teniendo que realizar en algunas ocasiones trabajos fuera del horario docente. De esta manera, la actividad afectará tanto a las clases prácticas como al periodo de trabajo autónomo que se le asigna al alumno fuera del aula. En la última clase del curso se realizará una sesión resumen en la que se expondrán los mejores resultados obtenidos en las prácticas realizadas.

Por otro lado, a lo largo del curso se llevarán a cabo encuestas a los alumnos para conocer su grado de satisfacción con el juego, así como para abordar la posibilidad de mejoras o detectar debilidades en su aplicación. Además, al final del curso se realizará un cuestionario transversal a todos los grupos para conocer el nivel de satisfacción global con la asignatura. La finalidad de esta encuesta es detectar si la gamificación supone o no una mejora en la implicación del alumno con la 
asignatura y supone un incentivo en su proceso formativo. En este mismo sentido, también se cruzarán las calificaciones de todos los grupos para conocer si el rendimiento es mayor o menor con la presencia de gamificación en la asignatura.

\section{Evaluación del proyecto}

Como se mencionó con anterioridad la gamificación propuesta tiene que ser evaluada tanto en sus inicios como a lo largo de su vida útil. Esto implicará que los juegos y dinámicas planteadas se enfrenten a modificaciones constantes. En los primeros pasos de GERENTUR se debe de marcar un grupo de control en la asignatura y otros en los que se emplee la gamificación. Para ello en ambos se aplica un modelo de evaluación de la satisfacción a través de escalas Likert en las que se evalúan el grado de motivación con los distintos aspectos de la asignatura como la comprensión, el contenido, o las dinámicas, entre otras. A los resultados obtenidos se les aplicará técnicas de estudio cuantitativas y cualitativas a través de programas como Stata o R-project. Estas consistirán en modelos relacionados con el análisis clúster, el cualitativo comparado (QCA - en inglés) o el de Componentes Principales (PCA - en inglés). Esto ayudará a ilustrar cómo se asocian los componentes del juego con las preferencias y motivaciones globales del alumno.

En este sentido Da Rocha, Gomes y Melo (2016) estudian la motivación a través de una serie de indicadores a los que les conceden una serie de niveles. Bajo esta estructura los autores realizan un análisis clúster en el que obtienen los distintos niveles de motivación de los distintos grupos que se les presentan en el aula. Por su parte De Marcos et al. (2016) emplean modelos de regresión y análisis de componentes principales para estudiar el efecto de la gamificación en entornos virtuales. Otra visión de la evaluación nos la ofrece el trabajo de Martínez, Fidalgo y Borrás (2016) en la que remarcan el uso del análisis cualitativo para evaluar la gamificación. Incluso aportan las entrevistas semiestructuradas como herramienta de evaluación.

Por otra parte, la adecuación de los juegos y dinámicas debe de estar siempre en constante evaluación para adaptarse a las nuevas necesidades docentes y laborales. Así, la constante extracción de información a los alumnos va a ser necesaria para lograr este objetivo. En este apartado, como se señaló anteriormente, se pueden emplear entrevistas semiestructuradas aplicadas a los alumnos o cuestionarios breves, abiertos y de escala, para detectar necesidades o evaluar la satisfacción de los participantes.

\section{Conclusiones y futuras extensiones}

La gamificación en el ámbito turístico está más estudiada desde el punto de vista de la comercialización de los servicios de este sector que desde la perspectiva docente ( $\mathrm{Xu}$, Weber y Buhalis, 2013; Sigala, 2015; Xu et al., 2016). Debido a esta circunstancia se debe de hacer una revisión previa a cómo se emplea la gamificación en el aula para después intentar implementarla en la docencia en turismo. Entonces, para su desarrollo hay que tener en cuenta los distintos tipos de alumnos que pueden comparecer en el aula (Tondello et al., 2016), presentar una historia atractiva (Kapp et al., 2013), fomentar una competencia "saludable" (Yu et al., 2002) y seguir las bases teóricas de incentivos - refuerzos (Richer et al., 2015). Con todo este aparato teórico se debe de empezar el diseño de los juegos a implementar teniendo en cuenta las características de la asignatura y los niveles motivacionales previos del alumno. No se debe de olvidar en este proceso que el alumno debe de tener la posibilidad de no participar sin menoscabar en su calificación o proceso formativo (Marache y Brangier, 2013). 
La técnica planteada en este trabajo se puede aplicar transversalmente a otras asignaturas relacionadas con los métodos estadísticos y/o numéricos tanto en el Grado en Turismo como en otros relacionados con las ciencias sociales. Además, la Narrativa planteada es fácil de adaptar a otras ramas académicas de las ciencias sociales como la economía, la empresa o las relaciones laborales, entre otras. Esto provoca que se pueda estudiar su incidencia desde múltiples aspectos y ver su efectividad desde diferentes problemáticas relacionadas con las estructuras de los planes de estudio. Por tanto, los presentes autores esperan que otros profesores emulen las ideas aquí expuestas, con las modificaciones necesarias para adaptarlo a otras áreas de las ciencias sociales. Esto permitiría comprobar si efectivamente las ideas fundamentales de la Narrativa aquí expuestas (pirámide laboral adaptada al sector profesional en cuestión, separación en fase individual y grupal, elección de rol dentro del grupo en función de la puntuación individual obtenida anteriormente) aumentan la implicación e interés del alumnado por una asignatura que a priori no forma parte de su núcleo de interés.

Por otra parte, se han de desarrollar estudios sistemáticos en los que se investigue desde un punto de vista cuantitativo la efectividad del sistema planteado. Por ello, se debe aprovechar dicha transversalidad para aplicarlo en otros planes de estudio, con lo que se podría observar la evolución de sus alumnos y el grado de implicación que registran con la asignatura. Esto permitiría desarrollar y mejorar el actual proyecto, así como contrastar sus beneficios más allá del plano teórico. En este sentido, es crucial la existencia de grupos de control con un mínimo de parámetros distintos, es decir, que de preferencia sean del mismo profesor, de la misma promoción, e incluso que hayan sido separados de forma aleatoria a partir de un grupo de mayor tamaño (Hanus y Fox, 2015). Esto permitiría realmente medir de forma objetiva el impacto de un proyecto de gamificación tal como lo hemos presentado aquí, no sólo para la motivación y satisfacción del alumno, sino también para sus resultados académicos, tal y como últimamente debería ser la ambición de cualquier proyecto de innovación docente.

\section{Agradecimientos}

Este trabajo se enmarca en el desarrollo del Proyecto 321 concedido en la Convocatoria de Proyectos de Innovación y Mejora de la Calidad Docente 2018/2019 que otorga la Universidad Complutense de Madrid. 


\section{Referencias}

Batlle, J. y González, V. (2017). Análisis de secuencias didácticas gamificadas para la enseñanza de lenguas extranjeras: la importancia de la narrativa en la gamificación. En Actas del $V$ Congreso Internacional de Videojuegos y Educación (CIVE'17). Disponible en: https://riull.ull.es/xmlui/bitstream/handle/915/6640/CIVE17_paper_4.pdf?sequence=1\&isAllowed=y

Cantador, I. (2015). La competición como mecánica de gamificación en el aula: Una experiencia aplicando aprendizaje basado en problemas y aprendizaje cooperativo. Gamificación en aulas universitarias, 67.

Cantador, I. y Conde, J. M. (2010). A Simple E-learning System based on Classroom Competition. En Proceedings of the 5th European Conference on Technology Enhanced Learning, 488-493.

Cárdenas, P.J., Pulido, J.I. y Carrillo, I. (2016). Adquisición de competencias en el Grado de Turismo mediante el aprendizaje basado en estudios de caso. Aula Abierta, 44(1), 15-22.

Castilla, G., Romana, M.G., y López-Terradas Aparicio, B. (2013). Concursando en el aula: la gamificación mediante quiz-show como herramienta de dinamización docente. Disponible en: https://abacus.universidadeuropea.es/handle/11268/2043

Contreras, R. S. (2016), Gamificación en aulas universitarias. Institut de la Cominicació. Universitat Autònoma de Barcelona.

Cortizo, J. C., Carrero, F. M., Monsalve, B., Velasco, A., Díaz, L. I., y Pérez, J. (2011). Gamificación y Docencia: Lo que la Universidad tiene que aprender de los Videojuegos. Disponible en: https://abacus.universidadeuropea.es/handle/11268/1750

Da Rocha, L., Gomes, A. S., y De Melo, I. J. (2016). Effectiveness of gamification in the engagement of students. Computers in Human Behavior, 58, 48-63.

De Marcos, L., García-López, E., García-Cabot, A., Medina-Merodio, J. A., Domínguez, A., Martínez-Herráiz, J. J., y Diez-Folledo, T. (2016). Social network analysis of a gamified e-learning course: Small-world phenomenon and network metrics as predictors of academic performance. Computers in Human Behavior, 60, 312-321.

Fitz-Walter, Z., Tjondronegoro, D., y Wyeth, P. (2011, Noviembre), Orientation passport: using gamification to engage university students. En Proceedings of the 23rd Australian computer-human interaction conference, $122-125$

González, C. (2016): Sistema de evaluación gamificada. En Espinosa, R. y Eguia, JL (Eds.). Gamificación en aulas universitarias. Insitut de la Comunicació. Universidad Autónoma de Barcelona.

Gomez, C.G. y De Pablos, C. (2013). The gamification and the enrichment of innovation practices in the firm: an analysis of experiences. Intangible Capital, 9(3), 800-822.

Hamari, J., Koivisto, J. y Sarsa, H. (2014). Does gamification work? --a literature review of empirical studies on gamification. In 47th Hawaii international conference on system sciences (HICSS), pp. 3025-3034.

Hofacker, C. F., De Ruyter, K., Lurie, N. H., Manchanda, P., y Donaldson, J. (2016), "Gamification and mobile marketing effectiveness". Journal of Interactive Marketing, 34, 25-36.

Hanus, M. D. y Fox, J. (2015). Assessing the effects of gamification in the classroom: A longitudinal study on intrinsic motivation, social comparison, satisfaction, effort, and academic performance. Computers \& Education, 80, 152-161.

Kapp, K., Blair, L. y Mesch, R. (2013), The gamification of learning and instruction fieldbook: Theory into practice. New York, NY: John Wiley \& Sons.

Kapp, K. (2012), The Gamification of Learning and Instruction: Game-based Methods and Strategies for Training and Education. New York, NY: Wiley \& Sons

Kokkalia, G., Drigas, A., Roussos, P. y Economou, A. (2017). Gamifying education: what is known, what is believed and what remains uncertain: a critical review. International Journal of Educational Technology in Higher Education, 14(1), 1-36.

Marache, C., y Brangier, E. (2013). Process of gamification. In Proceedings of the 6th Centric, 126-131.

Martínez, L. V., y del Moral, M. E. (2015). Gamificación: Estrategia para optimizar el proceso de aprendizaje y la adquisición de competencias en contextos universitarios. Digital Education Review, 27, 13-31.

Martínez, M., Fidalgo, Á., y Borrás, O. (2016). New challenges for the motivation and learning in engineering education using gamification in MOOC. International Journal of Engineering Education, 32(1B), 501512.

Miguel, S. E., Sorolla, G. R., Gato, M. P., y Guillem, J. A. (2017). Gamificación en Formación en Empresas. Dirección y Organización 62, 35-40. 
Mora, A., Riera, D., Gonzalez, C., y Arnedo-Moreno, J. (2015). A literature review of gamification design frameworks. In 7th International Conference on Games and Virtual Worlds for Serious Applications (VS-Games), 1-8.

O'Donovan, S., Gain, J., y Marais, P. (2013). A case study in the gamification of a university-level games development course. In Proceedings of the South African Institute for Computer Scientists and Information Technologists Conference, 242-251.

Pavía, J. M., Martínez, R., Morillas, F. G., y García, B. (2013). Docencia en Estadística. Experiencias de innovación. ADD Editorial. ISBN: 978-84-9858-872-9.

Richter, G., Raban, D. R. y Rafaeli, S. (2015). Studying gamification: the effect of rewards and incentives on motivation. En Richter et al., Gamification in education and business (pp. 21-46). Springer, Cham.

Rodríguez-Fernández, L. (2017). Smartphones y aprendizaje: el uso de Kahoot en el aula Universitaria. Revista Mediterránea de Comunicación, 8(1), 181-189.

Ryan, R. M., y Deci, E. L. (2000). Self-determination theory and the facilitation of intrinsic motivation, social development, and well-being. American Psychologist, 55, 68-78.

Prieto Martín, A., Díaz Martin, D., Monserrat Sanz, J., y Reyes Martín, E. (2014). Experiencias de aplicación de estrategias de gamificación a entornos de aprendizaje universitario. Revisión, 7(2).

Rodríguez, J. B., ARGUELLO, M., y Font, J. T. P. (2018). La narrativa como elemento cohesionador de tareas gamificadas para la enseñanza de lenguas extranjeras. RiMe, n. 2/II n. S., giugno 2018, 121-160. DOI $10.7410 / 1357$

Rodríguez, F. M., Vannasaeng, F., Esponda, S., Pasini, A. y Pesado, P. M. (2016). Flip-Flop: aplicación de buenas prácticas a partir de la gamificación. In Congreso Argentino de Ciencias de la Computación, 22. ISSN-e: 1989-1199,

Salcu, A. V., y Acatrinei, C. (2013). Gamification applied in affiliate marketing. Case study of Parale. Management \& Marketing, 8(4), 767-790.

Shindler, J. (2007). Transformative Classroom Management. Pearson Allyn \& Bacon Press.

Sigala, M. (2015). Gamification for crowdsourcing marketing practices: Applications and benefits in tourism. En Advances in crowdsourcing (pp. 129-145). Springer, Cham.

Todorov, T. (1969). Grammaire du Decameron. The Hague: Mouton.

Tondello, G. F., Wehbe, R. R., Diamond, L., Busch, M., Marczewski, A., y Nacke, L. E. (2016). The gamification user types hexad scale. In Proceedings of the 2016 annual symposium on computer-human interaction in play, 229-243.

Werbach, K., y Hunter, D. (2015). The gamification toolkit: dynamics, mechanics, and components for the win. Wharton Digital Press.

Xu, F., Tian, F., Buhalis, D., Weber, J., y Zhang, H. (2016). Tourists as mobile gamers: Gamification for tourism marketing. Journal of Travel \& Tourism Marketing, 33(8), 1124-1142.

Xu, F., Weber, J., y Buhalis, D. (2013). Gamification in tourism. In Information and communication technologies in tourism 2014 (pp. 525-537). Springer, Cham.

Yu, F. Y., Chang, L. J., Liu, Y. H., y Chan, T. W. (2002), Learning Preferences towards Computerised Competitive Modes. Journal of Computer-Assisted Learning, 18(3), 341-350. 